\title{
Augmentation du volume transversal par une ROG associée à du biomatériau en secteur esthétique après une greffe autogène. Case Report.
}

\section{Offerle JM'1, Sage $\mathrm{PO}^{2}$, Keller $\mathrm{P}^{1,2}$}

1. Hôpitaux Universitaires de Strasbourg (HUS) - France

2. Cabinet de Chirurgie Orale - Université Louis Pasteur - Strasbourg

\section{Introduction}

En chirurgie implantaire, un volume suffisant dans le secteur antérieur assure un meilleur soutien de la lèvre supérieure. Les différentes étapes d'une prise en charge implantaire (pré-implantaire, implantaire, et mise en condition) doivent se faire dans un souci permanent de gestion du résultat cosmétique.

L'objectif de cette présentation est de vous exposer un case-report sur l'utilisation d'une membrane (Symbios ${ }^{\circledR}$ ) associée à du biomatériau (Geistlich Bio Oss $®$ ), lors de la pose des implants dans un secteur greffé au préalable afin d'améliorer le pontique et de maximiser le soutien de la lèvre.

\section{Case-report}

Nous rapportons le cas d'une patiente de 37 ans et sans antécédents médico-chirurgicaux. Elle est adressée pour une prise en charge implantaire concernant le remplacement de son bridge maxillaire antérieur. Les examens clinique et radiographique révèlent la présence d'un défaut osseux important en regard de cette restauration prothétique. Quatre mois après avoir greffé la zone par de l'os autogène, l'intervention a consisté à poser deux implants associée à du biomatériau (sous forme de particule) stabilisé par une membrane lentement résorbable. En second temps chirurgical, un lambeau pédiculé palatin a été réalisé.

\section{Discussion}

La ROG est réalisée lors de la pose des implants car le biomatériau ne s'intègre pas à l'os greffé comme le démontre Cordaro et all. Cette ROG améliore le résultat cosmétique car il y a un meilleur soutient des tissus mous et protège la greffe (Luka et TUkel en 2013). Nous avons choisi une membrane résorbable car elle réduit la fréquence et la sévérité des complications par rapport à une membrane non résorbable (Proussaefs et Lozada 2003).

\section{Conclusion}

L'intérêt d'associer une ROG au moment de la pose des implants permet d'améliorer le support de la lèvre et du pontic. Ce cas montre que l'intégration du biomatériau devrait empêcher les changements de volumes osseux dans le temps et augmente le volume transversal.

dr.jeanmartin.offerle@gmail.com

(C) The authors, published by EDP Sciences. This is an Open Access article distributed under the terms of the Creative Commons Attribution License 4.0 (http://creativecommons.org/licenses/by/4.0/). 